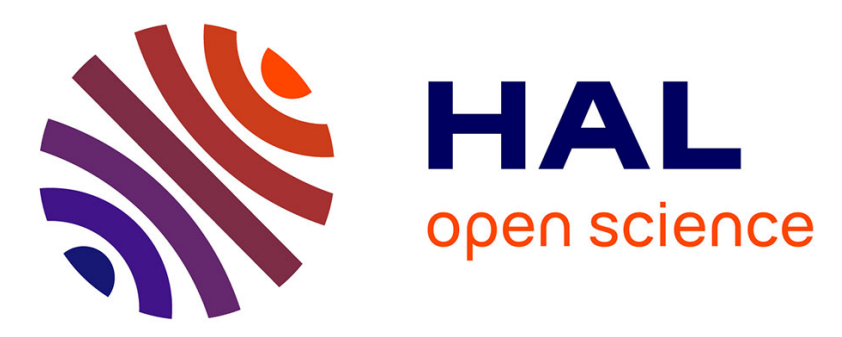

\title{
High-speed imaging optical techniques for shockwave and droplets atomization analysis
}

Pierre Slangen, Pierre Lauret, Frederic Heymes, Laurent Aprin, Nicolas

Lecysyn

\section{- To cite this version:}

Pierre Slangen, Pierre Lauret, Frederic Heymes, Laurent Aprin, Nicolas Lecysyn. High-speed imaging optical techniques for shockwave and droplets atomization analysis. Optical Engineering, 2016, 55 (12), pp.121706. 10.1117/1.OE.55.12.121706 . hal-01962648

\section{HAL Id: hal-01962648 \\ https://hal.science/hal-01962648}

Submitted on 20 Dec 2018

HAL is a multi-disciplinary open access archive for the deposit and dissemination of scientific research documents, whether they are published or not. The documents may come from teaching and research institutions in France or abroad, or from public or private research centers.
L'archive ouverte pluridisciplinaire HAL, est destinée au dépôt et à la diffusion de documents scientifiques de niveau recherche, publiés ou non, émanant des établissements d'enseignement et de recherche français ou étrangers, des laboratoires publics ou privés. 


\section{Optical Engineering}

\section{High-speed imaging optical techniques for shockwave and droplets atomization analysis}

Pierre R. Slangen

Pierre Lauret

Frederic Heymes

Laurent Aprin

Nicolas Lecysyn 


\title{
High-speed imaging optical techniques for shockwave and droplets atomization analysis
}

\author{
Pierre R. Slangen, ${ }^{\mathrm{a}, \star}$ Pierre Lauret, ${ }^{a}$ Frederic Heymes, ${ }^{a}$ Laurent Aprin, ${ }^{a}$ and Nicolas Lecysyn ${ }^{b}$ \\ aInstitute for Risk Sciences, LGEI, Ecole des Mines d'Alès, 30319 Alès Cedex, France \\ ${ }^{\mathrm{b}}$ CEA Gramat, BP80200, 46500 Gramat, France
}

\begin{abstract}
Droplets atomization by shockwave can act as a consequence in domino effects on an industrial facility: aggression of a storage tank (projectile from previous event, for example) can cause leakage of hazardous material (toxic and flammable). As the accident goes on, a secondary event can cause blast generation, impacting the droplets and resulting in their atomization. Therefore, exchange surface increase impacts the evaporation rate. This can be an issue in case of dispersion of such a cloud. The experiments conducted in the lab generate a shockwave with an open-ended shock tube to break up liquid droplets. As the expected shockwave speed is about $400 \mathrm{~m} / \mathrm{s}$ ( Mach 1.2), the interaction with falling drops is very short. High-speed imaging is performed at about 20,000 fps. The shockwave is measured using both overpressure sensors: particle image velocimetry and pure in line shadowgraphy. The size of fragmented droplets is optically measured by direct shadowgraphy simultaneously in different directions. In these experiments, secondary breakups of a droplet into an important number of smaller droplets from the shockwave-induced flow are shown. The results of the optical characterizations are discussed in terms of shape, velocity, and size. ๑ 2016 Society of Photo-Optical Instrumentation Engineers (SPIE) [DOI: 10.1117/1.OE.55.12.121706]
\end{abstract}

Keywords: high-speed imaging; shadowgraphy; particle image velocimetry; speckle; shockwave; droplets.

Paper 160554SS received Apr. 15, 2016; accepted for publication Jun. 1, 2016; published online Jun. 20, 2016.

\section{Introduction}

During industrial accidents involving successive explosions, flying fragments, shockwaves, and high heat radiation can occur. Loss of containment of the product can be generated while impacting a tank of low flammable and/or hazardous liquid (e.g., liquified natural gas, fuel, ammoniac, and so on) in conventional storage conditions. The leak is then ejected in the surrounding atmosphere as liquid jets, big droplets, or filaments. ${ }^{1}$ Krishna et al. ${ }^{2}$ mentioned that these droplets have important probabilities to disperse across a larger area than the liquid bulk. Moreover, the interaction of these two phase jets with shockwaves, coming from a secondary explosion (e.g., in domino effect), may reach the atomized droplets producing dispersion of a toxic or flammable cloud. These interactions are essential in risk sciences. ${ }^{3}$

A previous paper of the authors ${ }^{4}$ concerned the interaction of high-speed air flux with falling droplets of different products. In this study, time-resolved shadowgraphy with pulsed laser-induced fluorescence (PLIF) light source coupled with high-speed imaging camera was applied to characterize the interaction of the high-speed flow and the droplets at small scale [field of view (FOV): $20 \mathrm{~mm} \times 20 \mathrm{~mm}$ ]. The results showed the effect of the flow speed and the influence of the physicochemical properties of the droplets liquid. Conversely, for this paper, the ranges of flow speed and falling droplet size yield only to catastrophic breakup, whatever the product: Weber number is kept over 350 . The results of this present work can thus be extrapolated to other products if Ohnesorge number ${ }^{5}$ is kept inferior to 0.1 . Moreover, a brand new shockwave generator has been developed and equipped

*Address all correspondence to: Pierre R. Slangen, E-mail: pierre.slangen@ mines-ales.fr with high-speed sensors to record the pressure variations. These measurements are performed inside and outside the open-ended shock tube (OpEST). Moreover, dedicated pressure sensors have been placed to accurately determine the shockwave speed at different points.

From the optical point of view, the main problem for these applications is the frame rate of the imaging sensors to record the different stages of droplet evolution during the shock and also to fully characterize the shockwave in lateral extension. In Ref. 4, the authors used PLIF light source from a double cavity $15 \mathrm{~mJ}-10 \mathrm{~ns}$ pulsed Nd-YLF laser impinging doped polymer to generate a 10 -cm-diameter collimated beam of yellow incoherent light. This system can deliver double pulse of up to $15 \mathrm{~mJ}$ at $4.5 \mathrm{kHz}$ synchronized with high-speed imaging camera in particle image velocimetry (PIV) mode. The objects are then "frozen" during the pulse time (10 ns) and two successive pulses can be recorded within the straddle time of the high-speed sensor (e.g., $\sim 200 \mathrm{~ns}$ ). The full frame throughput of the camera is then divided by the number of images related to the number of pulses. For higher frame rates, superluminescent LED arrays can deliver up to 15,000 lumens continuous wave and freezing the object is then reached up to $290 \mathrm{~ns}$ by camera electronic shuttering. Shadowgraphy implies parallel beams or collinear beams between the lighting source and observation sensor. This strongly affects the range of observations to some tens of centimeters if large and high-cost lenses or mirrors have to be avoided. Some authors presented high-speed Edgerton retroreflective shadowgraphy ${ }^{6}$ coping with large-scale measurement of explosions or gunshots. ${ }^{7-9}$ For simplicity reason, we now call it pure in line shadowgraphy (PILS). Finally,

0091-3286/2016/\$25.00 @ 2016 SPIE 
local shadowgraphy in the vicinity of the droplets and PILS enable a jump in the magnification range to collect information with temporal and spatial resolutions. It is a kind of multiplexing the recording system thanks to two high-speed imaging cameras (7000 fps, 12 bits, $1280 \times 960$ pixels) for shadowgraphy, the third one for PILS (2000 fps, 12 bits, $1024 \times 1024$ pixels), and high-speed sensors (pressure and temperature) to fully determine the physical parameters.

\section{Material and Methods}

This section will describe the setup and mainly focus on the shock tube. The principle of the different optical techniques will then be detailed and also the optical sensors commonly coupled with different kinds of lightings, e.g., LEDs, laser, or metal vapor lamps.

\subsection{Open-Ended Shock Tube}

The device generating the shockwave is made of steel with an inside diameter of $15 \mathrm{~cm}$ (Fig. 1). The whole tube is about $2.36 \mathrm{~m}$ and divided into three pieces (high pressure chamber or driver section, rupture disk, and driven section). Driver section is $30-\mathrm{cm}$ long and separated from driven section by a rupture disk made of 2/10-mm thick aluminum calibrated to ruin at driver gauge pressure of 4.2 bars. Driven section is thus hermetically linked to a 2-m long parallel tube. The droplet generator is placed at the exhaust so the drops are free falling in front of the shockwave. The droplet generator is built with an automatic syringe pusher linked to a soft tube ended with calibrated injection needle. Inside the driver section, temperature and static pressure are recorded. Four highfrequency pressure sensors (three PCB model 102A06 and one model 137A23) are set to record the shockwave. These sensors are connected to fast data acquisition HBM GEN7T, synchronizing all data, image acquisition, and triggering (peak detection from pressure sensors, external sound trigger, or manual trigger) at $250-\mathrm{kHz}$ sampling.

Pressure sensor C1-28810 is fed directly in the tube nearby the inner surface and gives the pressure variation just before the tube outlet. Pressure sensors C2-28018 and C328016 are placed on the same free-field blast pressure rod and separated by $100 \mathrm{~mm}$ (blast gage). This homemade configuration rod enables measuring the exact speed of the shock at $310 \mathrm{~mm}$ of the tube outlet. The rod has been machined to avoid any perturbation of the shock displacement. Sensor C4-7074 is placed on a single rod and measures the reduced field pressure at $5 \mathrm{~m}$ of the tube outlet. First tests have been carried out to determine safety distance for optics and cameras.

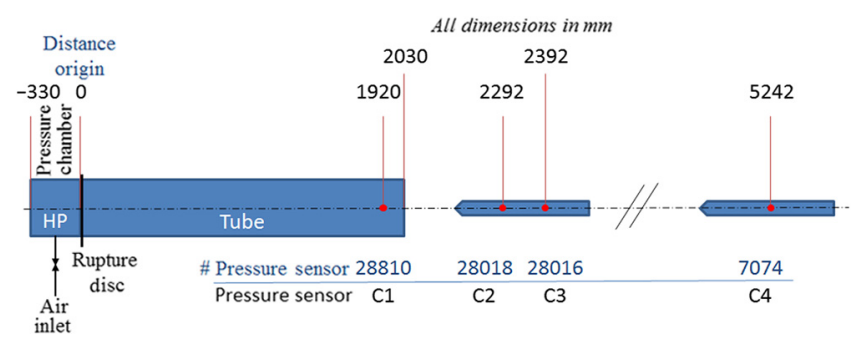

Fig. 1 Shock tube configuration with sensors.

\subsection{Optical Techniques}

Nowadays, optical techniques dedicated to detect or to measure refractive index variations ${ }^{7}$ can be coupled with highspeed imaging. This combination leads to time-resolved measurements of the refractive index change. This change can then be used to track velocity or shape of, e.g., shockwave and particles.

For PIV, the medium under study is seeded with particles. These particles are then diffusing/reflecting the impinging light (typically from LEDs or laser with light sheet generator). The high-speed camera is then focused on the light sheet and particle movement is recorded. Afterward, particle cluster is tracked by digital image correlation (DIC) algorithms to retrieve displacement or speed maps (e.g., Dantec

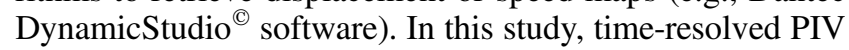
(TRPIV) will be applied to characterize the flow behind the shockwave by seeding the outlet shock tube with smoke particles. The laser sheet is horizontal and the camera observation axis is vertical (not shown for simplicity on Fig. 2). After the flow study, the droplet generator is combined to the presented setup.

Then, for accurate measurement of droplets shapes, direct shadowgraphy is completed using backlighting of the droplets with collimated light beam from superlum LED array coupled with 12 bits Phantom V711 (V711S, S for Shadow) with Tamron 70 to $300 \mathrm{mmf} / 4$ to 5.6 zoom lens. Particles tracking or PIV algorithms can then be applied to the whole drop or to secondary droplets after atomization.

As mentioned above, we have chosen to gently rename retroreflective Edgerton shadowgraphy into PILS. Hence, the acronym is closer to (TR-SR) PIV, PLIF, laser-induced fluorescence (LIF), DIC, and so on and also enables us to acknowledge "Doc Edgerton" giving the right pill removing the researcher's headache while chasing after large-field shadowgraphy solutions. The final collinear solution has been carried on by Settles et al. ${ }^{8}$

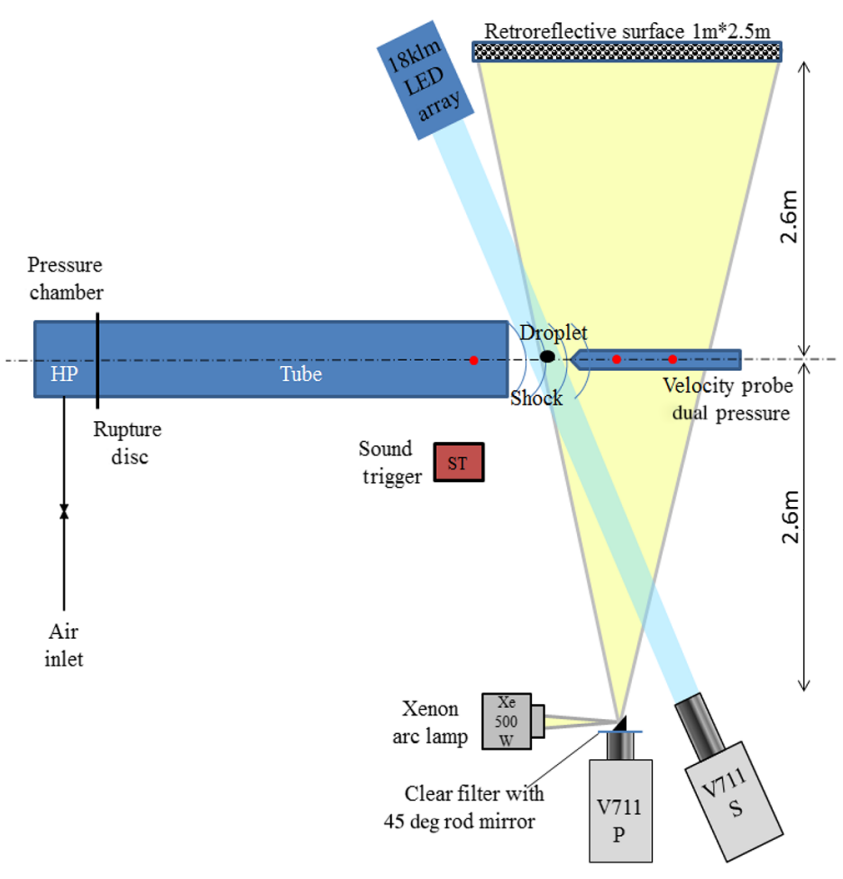

Fig. 2 Shockwave tube and optical setups: PILS and direct shadowgraphy (PIV not shown). 


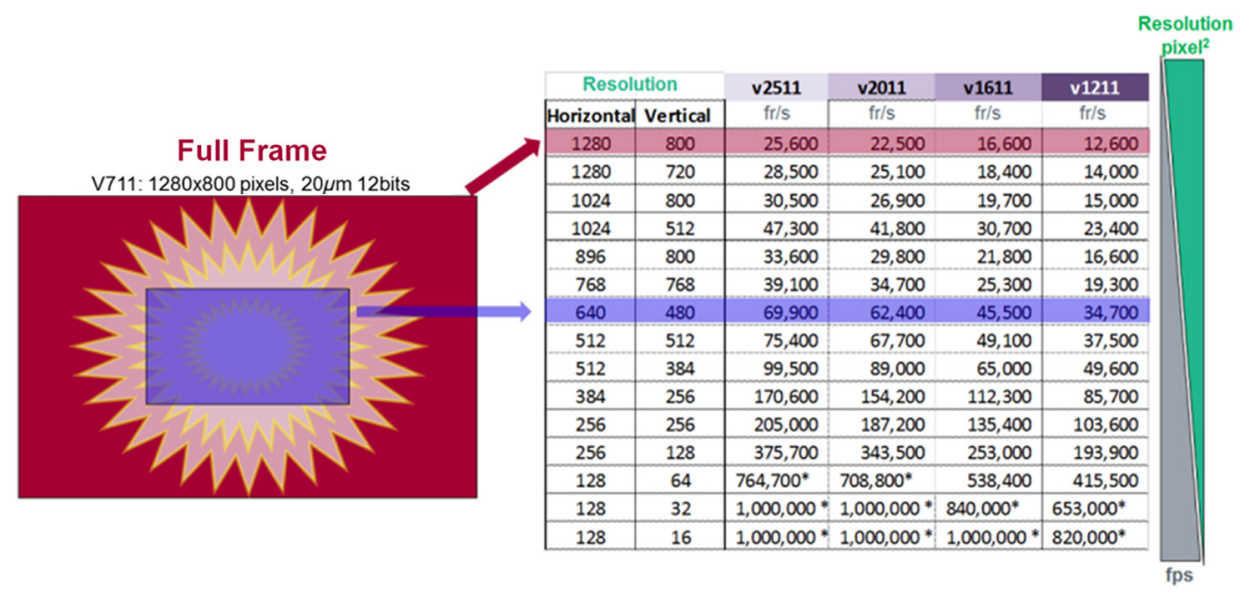

Fig. 3 Effect of reduced resolution on the effective FOV and recording frequency.

The basics are to use retroreflective screen to get every illuminating beam backreflected in the same direction and so collinear even in case of diverging illumination for a given angle range. The trick is then to place a small 45-deg rod mirror cemented at the center of the clean filter mounted on the imaging optics. The point source is then focused on the mirror. Therefore, the centers of illumination and of observation diverging beams are perfectly matched. Moreover, when the distances between the camera-refractive object and refractive object-retro reflective screen are equivalent, the object and its shadow are then perfectly superimposed.

To cope with large FOV, PILS setup is integrated in the same area to get droplet view and shock propagation (Fig. 2). Therefore, the second Phantom V711 camera (V711P, P for PILS) is used to record the PILS signal from the retroreflective surface (Orafol, Oralite5300 Reflective Film). This adhesive film is made of catadioptric glass beads embedded in a transparent layer of plastic material. The specific coefficient of retroreflection $R^{\prime}$ is about $50 \mathrm{~cd} / 1 \mathrm{x} / \mathrm{m}^{2}$ for $0.2-\mathrm{deg}$ angle of observation and entrance angle less than $5 \mathrm{deg}$.

Finally, for synchronized multiview in high speed, ${ }^{10,11}$ two Phantom V711 cameras have been used for shadowscopy (side view and top view) combined with superlum LED arrays. Photron SA3 (2000 fps, 12 bits, $1024 \times 1024$ pixels has been used for the PILS.

\subsection{High-Speed Cameras}

For high-speed imaging, ${ }^{12}$ CMOS rolling memory buffer (RMB) and in situ image storage (ISIS) sensors (CCD or $\mu \mathrm{CMOS}$ ) with local image storage are commonly used. ISIS sensors are more dedicated to ultrahigh-speed imaging (200 Mfps but only 200 stored full frames), while RMB CMOS is convenient for huge memory (up to $188 \mathrm{~GB}$ on-board memory) at lower, but full frame, frequencies (up to $26 \mathrm{kfps}$, 12 bits $1280 \times 960$ pixels). RMB CMOS can also reach a million fps at decreased resolution, as throughput is the bottleneck (for now, about 25 Gpixel/s for Phantom V2512 camera).

As mentioned in Sec. 2.1, different CMOS RMB cameras (available in our lab) have been used. Vision Research Phantom V711 cameras enable high-speed imaging at $22 \mathrm{kfps}$ for $320 \times 960$ pixels reduced resolution, and Photron SA3 reaches up to $6000 \mathrm{fps}$ for $300 \times 1024$ pixels reduced resolution. While working at reduced resolution, the effective
FOV is also reduced and this affects the choice of the lens to fully image the scene (Fig. 3).

These cameras have been coupled with lenses so as to catch the region of interest while preserving the optics safety. The minimal safety distance is about $1.5 \mathrm{~m}$ and such a long distance that macrophoto lenses have been used for the direct shadowgraphy of the droplets. For PILS, the lens aperture must fit with the rod mirror diameter $(4 \mathrm{~mm})$ to avoid center image blur. All dimensions in pixels have been transformed in real-space distances using calibration targets for the different ranges of measurements.

\subsection{Light Sources}

In high-speed imaging, short exposure time to freeze the object movement is the major rule. But rule number one is really "don't fry the object." In classical imaging, some black carbon-reinforced objects can rise up to $80^{\circ} \mathrm{C}$ in less than $60 \mathrm{~s}$ when lit by powerful $4 \mathrm{~kW}$ Hydrargyrum (mercury) medium-arc iodide spotlights. These stage spotlights are now commonly replaced by super bright LEDs (SB LED) gratings reaching up to 18 klumen of "cold" light for only $300 \mathrm{~W}$ consumption (e.g., Veritas Constellation 120). $\mathrm{Xe}$ or $\mathrm{Xe}-\mathrm{Hg}$ arc lamp is also applied when a high-power point source is required (e.g., for point-source schlieren or PILS).

For dedicated imaging like shadowgraphy and schlieren, SB LEDs are also used and will progressively replace pulsed laser for low-pulsed rates. On the other hand, pulsed laser is now reaching $20-\mathrm{kHz}$ rates of $100-\mathrm{mJ}$ pulse in the green line $(527 \mathrm{~nm}$ fd-NdYLF or $532 \mathrm{~nm} \mathrm{fd-NdYVO}$, where fd denotes the frequency doubling). Double or triple pulses can be generated by associating multiple cavities and triggering the laser emission with respect to the optical sensor head. Speckle is then generated in high-resolution imaging using lasers. Even if PIV lasers have bad light coherence, the speckle always appears. Therefore, these lasers are very often coupled with dyes-doped polymer plates and so generate LIF when lit by the laser beam. This contributes to reducing the speckle effect and insures image sharpness free of speckles and secondary interference fringes ${ }^{13}$ when using a Fresnel collimator or light sheet generator. With very short pulse duration and PIV mode for camera acquisition (the camera electronic shutter is kept "open" within the frame rate 


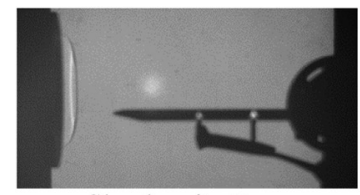

Shock exit: $T_{0}$

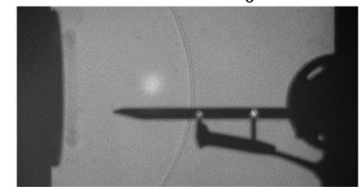

$T_{0}+5 \times 10^{-4} \mathrm{~s}$

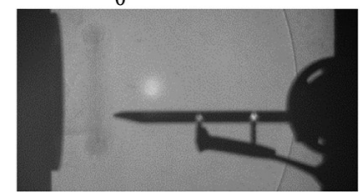

$T_{0}+10 \times 10^{-4} \mathrm{~s}$

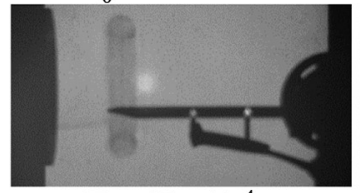

$T_{0}+15 \times 10^{-4} \mathrm{~s}$

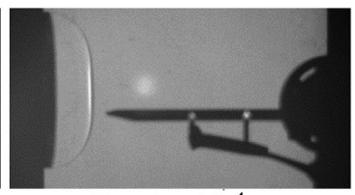

$T_{0}+1 \times 10^{-4} \mathrm{~s}$

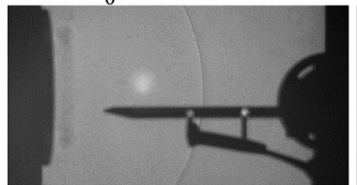

$T_{0}+6 \times 10^{-4} \mathrm{~s}$

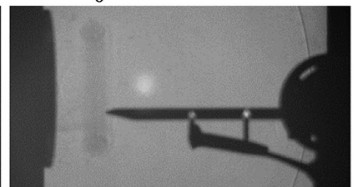

$T_{0}+11 \times 10^{-4} \mathrm{~s}$

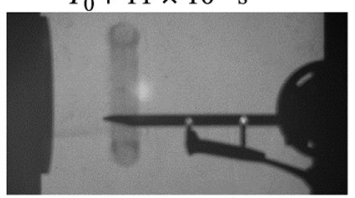

$T_{0}+16 \times 10^{-4} \mathrm{~s}$

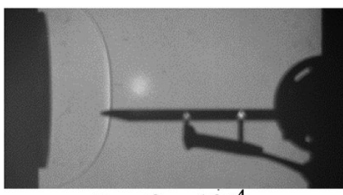

$T_{0}+2 \times 10^{-4} \mathrm{~s}$

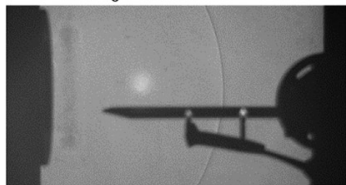

$T_{0}+7 \times 10^{-4} \mathrm{~s}$

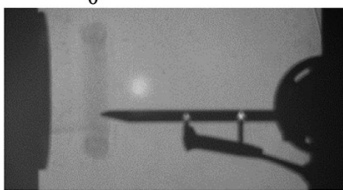

$T_{0}+12 \times 10^{-4} \mathrm{~s}$

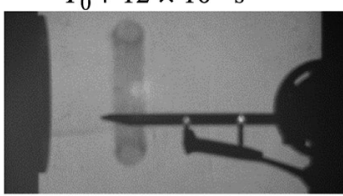

$T_{0}+17 \times 10^{-4} \mathrm{~s}$

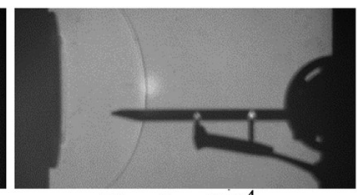

$T_{0}+3 \times 10^{-4} \mathrm{~s}$

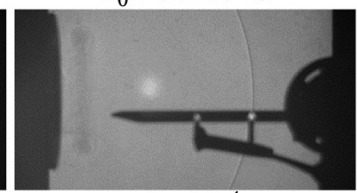

$T_{0}+8 \times 10^{-4} \mathrm{~s}$

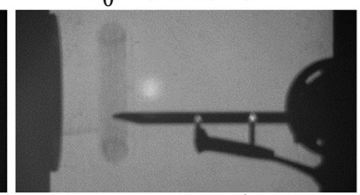

$T_{0}+13 \times 10^{-4} \mathrm{~s}$

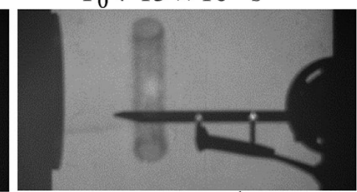

$T_{0}+18 \times 10^{-4} \mathrm{~s}$

Fig. 4 Shockwave propagation and vortex (FOV $630 \mathrm{~mm} \times 336 \mathrm{~mm}$ ).

and the pulse duration is the exposure time), lasers remain the best source to freeze the object movement.

\section{Results and Discussion}

This section will describe the results obtained for the shockwave characteristics and the interaction of this reproducible shockwave with falling droplets.

\subsection{Shockwave Characteristics}

The shockwave can be described in terms of velocity and geometry. The geometry is evolving during propagation from the outlet. Different tests have been carried out to select the right membrane configurations (aluminum thickness). Figure 4 presents the main phases of the wave evolution by PILS. The FOV is about $630 \mathrm{~mm} \times 330 \mathrm{~mm}$. On the left, the tube outlet is well contrasted from the reflecting screen. The shadow of the "homemade" double blast gage is clearly present. Two bright spots (retroreflective stickers) indicate the location of each high-speed blast gage separated by $100 \mathrm{~mm}$. The first gage is located $310 \mathrm{~mm}$ in front of the outlet. From the blast gages, connection wires are shielded and glued to the main pole stand. Plane wave (one dimension) from the inner tube $\left(T_{0}\right)$ is diffracted and evolves as spherical wave (three dimension). The vortex ring exits the tube $0.5 \mathrm{~ms}$ after the first shock $\left(T_{0}+5 \times 10^{-4} \mathrm{~s}\right)$ and propagates at about $165 \mathrm{~m} / \mathrm{s}$. The vortex ring presents a very good contrast at $T_{0}+17 \times 10^{-4} \mathrm{~s}$ as it is centered in the PILS setup. Shock velocity is around $436 \mathrm{~m} / \mathrm{s}$ and decreases to $360 \mathrm{~m} / \mathrm{s}$ after sensor C3 (Fig. 5).

An important decrease in overpressure and shock velocity can be observed at the exit of the OpEST. In order to characterize precisely the flow impacting the droplets, TRPIV has been used by coupling the double-cavity ND-YLF $15 \mathrm{~mJ}-4 \mathrm{kHz}$ with the V711 in PIV mode. Time between pulse is set to $20 \mu \mathrm{s}$. Seeding is done by smoke dust in the outlet tube. Velocity of the flow is measured from 200 to
$300 \mathrm{~m} / \mathrm{s}$ at the distance of falling droplets from the exit of OpEST. Figure 6 illustrates the sudden increase of the velocity field behind the shockwave. Time measurement starts when the shockwave exits the shock tube.

\subsection{Shockwave and Droplets Interaction}

As discussed in Sec. 2.2, the two fastest cameras Phantom V711 are focused on droplet measurements while the Photron SA3 records PILS.

Different sizes of droplets (diameter between 0.8 and $1.47 \mathrm{~mm}$ ) have been used but only one will be presented for the sake of simplicity. Using different droplet sizes and products also enables us to get a comparison with theoretical models. ${ }^{14}$ Figure 7 presents the comparison of global view (PILS) and lateral direct shadowgraphy in the rectangular "zoomed" area for two different time codes (dimensions in $\mathrm{mm}$ ).

The effect of dual lighting of the scene is clearly seen as atomized droplets in the PILS image are brightened after the interaction of the drop with the vortex ring. The secondary droplets cloud speed is around $150 \mathrm{~m} / \mathrm{s}$.

In the focused lateral view, primary tests have been conducted with drops around $3 \mathrm{~mm}$ in diameter and even the

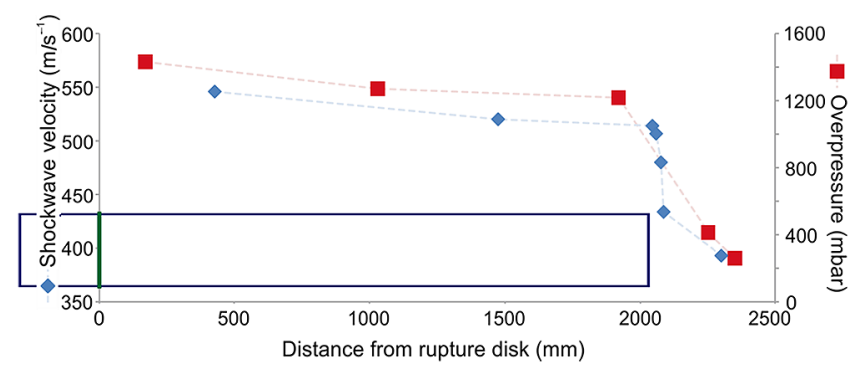

Fig. 5 Shock tube velocity and overpressure over distance from rupture disk. 


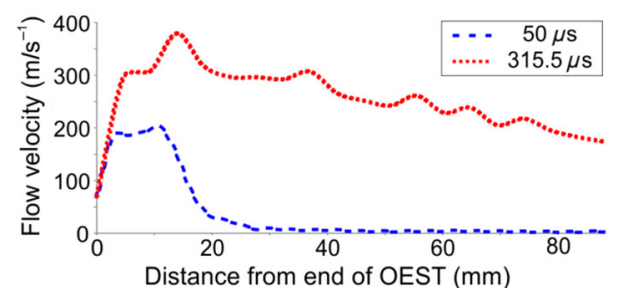

(a)

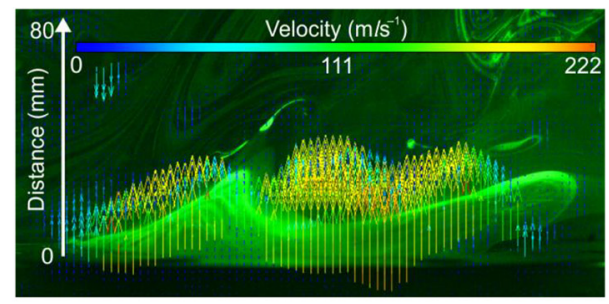

(b)

Fig. 6 (a) Flow velocity at different times after exit from OpEST, and (b) PIV analysis extraction at $50 \mu \mathrm{s}$.
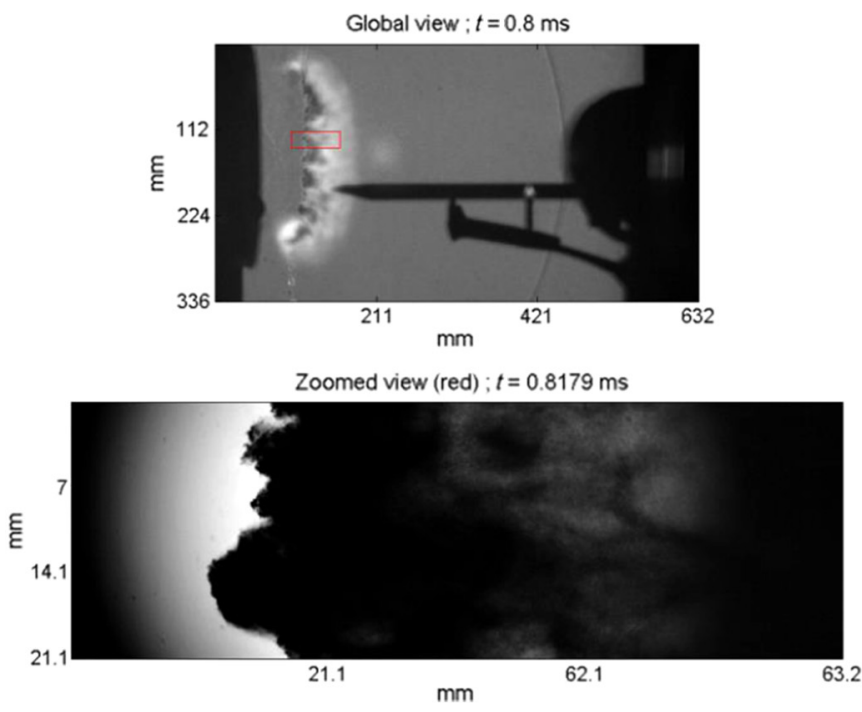

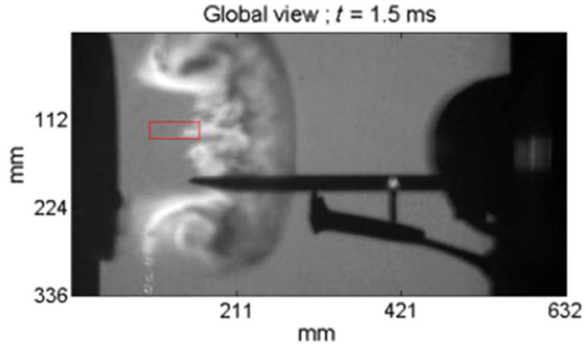

Zoomed view (red) ; $t=1.5 \mathrm{~ms}$

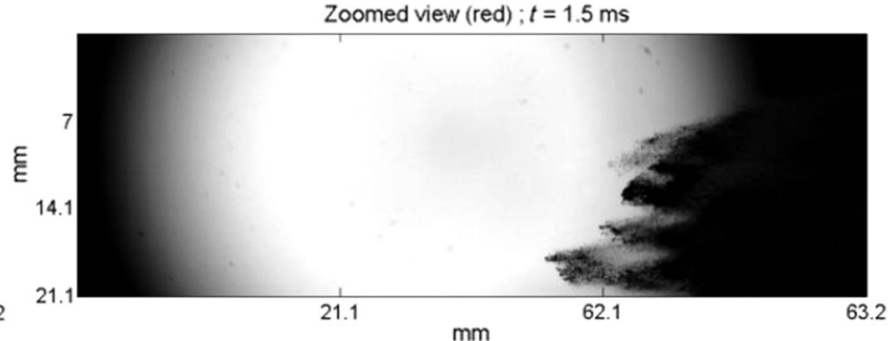

Fig. 7 Comparison of global and zoomed views at $t=0.8 \mathrm{~ms}$ and $t=0.15 \mathrm{~ms}$.

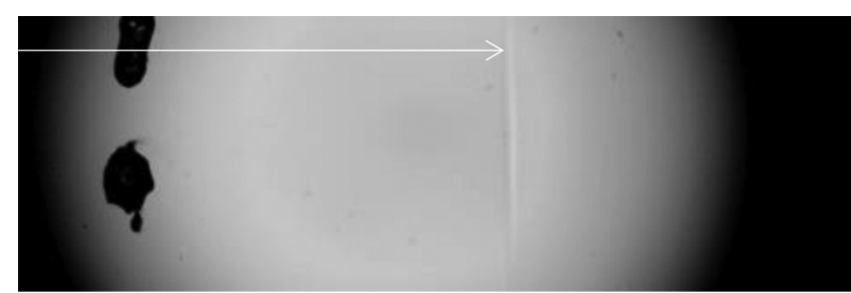

Fig. 8 Shockwave detected by direct shadowgraphy in zoomed view. shock is detected at $22 \mathrm{kfps}$ (Fig. 8, white arrow). The drop dispensing has been reduced to reach smaller droplets to ensure fewer secondary droplets after atomization.

The analysis of four droplets from $0.8 \mathrm{~mm}$ to $1.47 \mathrm{~mm}$ shows a catastrophic breakup with Weber number from 1191 to 2679. Figure 9 shows the images of a $0.8-\mathrm{mm}$ droplet fragmentation taken with a time step of $45 \mu \mathrm{s}$ (FOV $40 \mathrm{~mm} \times 30 \mathrm{~mm}, 22,000 \mathrm{fps}$ ). Dimensionless time ${ }^{14}$ is displayed on each picture. Total breakup time is reached at $T=5.5$.

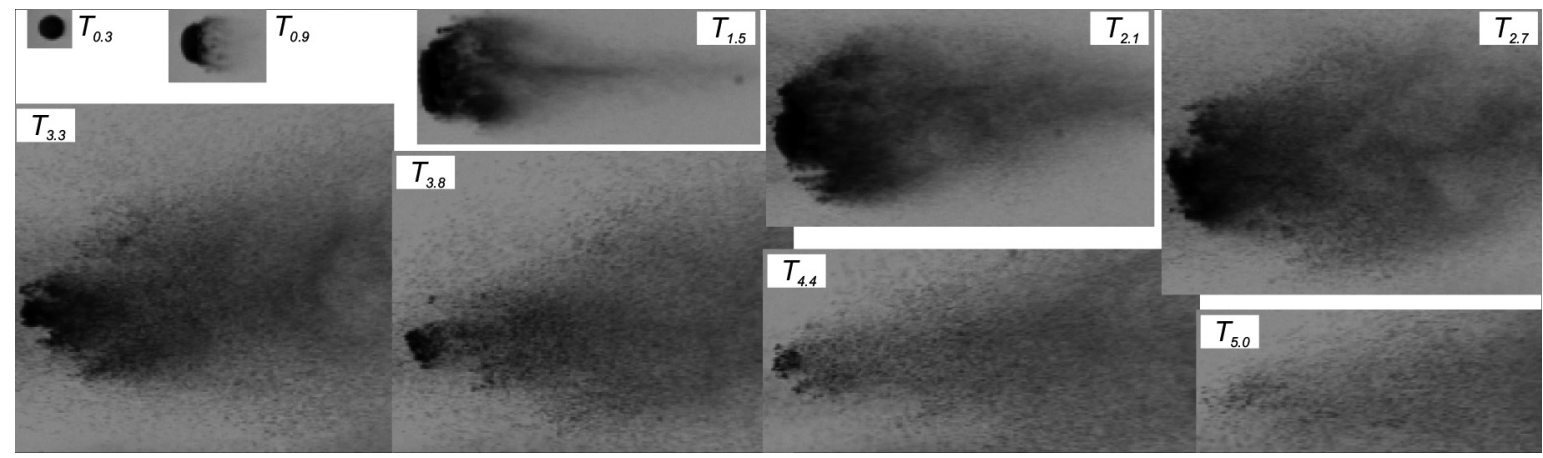

Fig. 9 Fragmentation of a 0.8-mm diameter droplet at different dimensionless times: compiled view. 

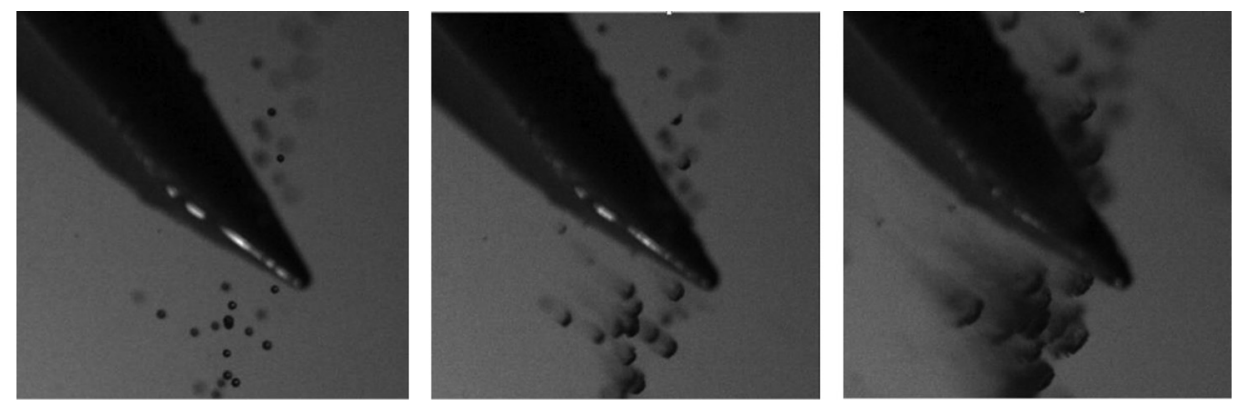

Fig. 10 Top views of $0.8-$ to $1.47-\mathrm{mm}$ droplets falling before the pressure transducer tip (full FOV).

First image is grabbed when the shockwave reaches the droplet. On the first images, the droplet is deformed by the flow and stripping is beginning on the sides. Then small perturbations appear on the windward surface of the drop. Finally, large amplitude surface waves penetrate the droplet, also observed in the literature. ${ }^{15,16}$ The wake of the drop is composed of a mist with droplet sizes less than the pixel dimension $(61 \mu \mathrm{m})$. The top view analysis confirms these measurements and also shows the extent of the mist cloud at 90 deg (Fig. 10).

To preserve the optics from the shock, the minimal working distance is about $1.5 \mathrm{~m}$. At this distance, even while using a long range optical microscope (e.g., Questar FR I MK III), it is not possible to optically resolve the secondary droplets as the pixel size is about $26 \mu \mathrm{m}$. Therefore, fragmentation droplets will be evaluated by a phase Doppler analyzer in future work.

\section{Conclusion}

This paper presents the interaction mechanisms of shockwaves interacting with falling droplets, as in the case of industrial accidents presenting liquid leaks submitted to shockwave from near-field secondary explosions. At the laboratory scale, we have adapted, to high-speed imaging, optical techniques sensitive to refractive index variations (and more precisely to the second derivative in case of direct shadowgraphy) to characterize the shockwave. This shockwave leaves from OpEST at about Mach 1.25. Immediately after the shock, flow velocity is increased and strongly interacts with drops. The velocity field following the shockwave has been studied with PIV using laser speckle generated from double-pulsed laser on smoke-seeded medium (air in our case). The direct displacements of the air have been used to compare the secondary droplets prediction with Pilch and Erdman models. Therefore, direct shadowgraphy in lateral and top views presents the drop atomization in very slow motion thanks to 22-kfps recordings. These optical configurations are really adapted to such measurements but have a little lack of extended range. Hence, PILS is performed to ensure large FOV and high-speed recording. Summing these techniques together enables us to fully understand the mechanism of atomization.

Future works will focus on direct Z-type shadowgraphy with large parabolic mirrors (30-cm diameter) coupled with high-speed pulsed laser $(20 \mathrm{kHz})$ and cameras. Higher sensitivity to refractive index variations will be performed by coupling simultaneously schlieren cutting edge observation in the same Z-type setup. This will also permit us to detect evaporation of some volatile liquid drops. Considering the safety distances for the optics, as the requested optical resolution (less than $15 \mu \mathrm{m} /$ pixel) is not reached by a classical imaging system, phase Doppler particles dynamics analysis will be evaluated for high-speed secondary tiny droplets after atomization.

\section{Acknowledgments}

This research was supported by the French Alternative Energies and Atomic Energy Commission (CEA, Commissariat à l'énergie atomique et aux énergies alternatives). Part of this work has been presented during the 10th Pacific Symposium on Flow Visualization and Image Processing, Naples, Italy, June 15-18, 2015.

\section{References}

1. N. Lecysyn et al., "Ballistic impact on an industrial tank: study and modeling of consequences," J. Hazard. Mater. 172(2-3), 587-594 (2009).

2. K. Krishna et al., "The use of aerosol formation, flammability, and explosion information for heat-transfer fluid selection," J. Hazard. Mater. 104(1-3), 215-226 (2003).

3. N. Lecysyn et al., "Preliminary study of ballistic impact on an industrial tank: projectile velocity decay," J. Loss Prev. Process Ind. 21(6), 627-634 (2008).

4. P. Slangen et al., "Blast wave interaction with droplets: time resolved measurements by laser shadowscopy," Proc. SPIE 8413, 841312 (2012).

5. L.-P. Hsiang and G. M. Faeth, "Near-limit drop deformation and secondary breakup," Int. J. Multiphase Flow 18, 635-652 (1992).

6. H. E. Edgerton, "Shock wave photography of large subjects in daylight," Rev. Sci. Instrum. 29, 171-172 (1958).

7. G. S. Settles, Schlieren and Shadowgraph Techniques: Visualizing Phenomena in Transparent Media, Springer-Verlag Berlin Heidelberg (2001).

8. G. S. Settles et al., "Full-scale high-speed "Edgerton" retroreflective shadowgraphy of explosions and gunshots," in Proc. of the 5th Pacific Symp. on Flow Visualization and Image Processing, Australia (2005).

9. D. Laboureur, J.-M. Buchlin, and P. Rambaud, "Small scale experiments on boiling liquid expanding vapor explosions: supercritical BLEVE," presented at Proc. ASME 2012 Pressure Vessels and Piping Conf., Volume 9: Rudy Scavuzzo Student Paper Symp. and Competition, 15-19 July 2012, Toronto, Ontario, Canada, Paper No. PVP2012-78283, pp. 51-60, Pressure Vessels and Piping Division.

10. P. L. Reu and T. J. Miller, "Synchronization errors in high-speed digital image correlation," in Proc. SEM (2009).

11. T. Siebert et al., "High-speed digital image correlation: error estimations and applications," Opt. Eng. 46(5), 051004 (2007).

12. S. F. Ray, High Speed Photography and Photonics, SPIE Press Bellingham, Washington (1997).

13. J. C. Dainty et al., Laser Speckle and Related Phenomena, 2nd ed., pp. 154-170, Springer Verlag, Berlin (1984).

14. M. Pilch and C. A. Erdman, "Use of breakup time data and velocity history data to predict the maximum size of stable fragments for acceleration-induced breakup of a liquid drop," Int. J. Multiphase Flow 13, 741-757 (1987)

15. C. H. Lee and R. D. Reitz, "An experimental study of the effect of gas density on the distortion and breakup mechanism of drops in high speed gas stream," Int. J. Multiphase Flow 26, 229-244 (2000).

16. D. D. Joseph, J. Belanger, and G. S. Beavers, "Breakup of a liquid drop suddenly exposed to a high-speed airstream," Int. J. Multiphase Flow 25, 1263-1303 (1999). 
Pierre R. Slangen received his $\mathrm{PhD}$ in physics from the University of Liege, Belgium, in 1995. He is a professor at the Ecole des mines d'Ales, France, and the head of the Risk Sciences Institute. For 20 years, he mainly worked on interferometric, speckle, and photographic techniques. He is involved in coupling holographic and speckle techniques with high-speed imaging for time-resolved measurements. Open-air large-scale measurements of refractive index are also carried out by shadowgraphy and background-oriented schlieren (BOS).

Pierre Lauret received his $\mathrm{PhD}$ in environment from Ecole des mines de Saint-Etienne, France, in 2014. He is an assistant professor at the Ecole des mines d'Ales, France, at the Risk Sciences Institute. His work is focused on the understanding of physical phenomena involved in industrial risks based on experimental studies. The phenomena involved are atmospheric dispersion resulting from accidental release, droplet fragmentation by overpressure wave, unconfined vapor cloud explosion, and so on.

Frederic Heymes is an engineer and received his $\mathrm{PhD}$ in chemical engineering from University of Montpellier, France, in 2005. He is a professor at the Ecole des mines d'Ales, France. For 10 years, he has developed his research in the field of industrial risks (fire, explosion, and atmospheric dispersion) with a specific focus on large-scale experiments. The objective of this research is to model the mechanisms and consequences of the phenomena and develop strategies to mitigate the effects, involving fluid mechanics and thermic skills. Now, he is a coordinator of the risks physics research team.

Laurent Aprin is a senior researcher in fluid mechanics and transfer. His research focused on thermohydraulics phenomena and twophase flow in industrial processes. Since 2005, he has worked at the Ecole des mines d'Ales as a professor on the evaluation and modeling of consequences of chemical spills in marine environment. His current research topics are related to the characterization of consequences of industrial accidents. He particularly studies the flow behaviors of chemicals, oil, and gas in the deep sea (shipwreck and offshore accidents).

Nicolas Lecysyn received his $\mathrm{PhD}$ in physics from the University of Provence, France, 2008. He is a researcher at Commissariat à I'Energie Atomique, France, and belongs to the energetic compositions efficiency laboratory. For 10 years, the common thread of his work has been industrial storage vulnerability versus explosive or ballistic aggression. As a consequence, optical diagnostic techniques (BOS, shadowgraph, PIV, and so on) are the main way to get qualitative and quantitative information in tests series led by him and his partners. 